\title{
Las personas mayores durante la pandemia COVID-19: políticas públicas y acceso a las tecnologías de la información y comunicación en Argentina
}

\section{Julieta Oddone}

(iD) https://orcid.org/0000-0002-2908-0046

CONICET, Argentina

FLACSO, Argentina

julietaoddone9@gmail.com

\section{Paula Pochintesta}

iD https://orcid.org/0000-0002-1455-8852

CONICET - Centro del Adulto Mayor "Mario Strejilevich", Instituto de Salud Colectiva, Departamento de Salud Comunitaria, Universidad Nacional de Lanús, Argentina ppochintesta@gmail.com

RESUMEN

Las personas mayores se encuentran insertas en una sociedad caracterizada por la aceleración tecnológica, la comunicación y la información. Estas transformaciones cambian a un ritmo vertiginoso, generando gran impacto en la vida de los mayores. Partimos de considerar a las personas mayores como sujetos activos y en pleno ejercicio de sus derechos, donde la inclusión digital forma parte la inclusión social. Así, el objetivo de este trabajo es, por un lado, realizar un análisis de contenido de las políticas implementadas en Argentina en materia de inclusión digital, lo que incluye el nivel de cobertura y relevamiento de posibles brechas existentes. Por otro lado, se analizan datos de una encuesta nacional sobre acceso a recursos y uso de tecnologías de información y comunicación por parte de las personas mayores. Las conclusiones muestran que existen brechas importantes tanto en el acceso a recursos como en la frecuencia de uso. Sobre las políticas públicas nacionales 
en la materia, observamos que la pandemia evidenció que no hubo un plan estratégico para el logro de una política de inclusión de las personas mayores a las nuevas tecnologías.

Palabras clave: personas mayores, TIC, Argentina, politicas públicas, programas.

\title{
Older people during the COVID-19 Pandemic: public policies and access to information and communication technologies in Argentina
}

\author{
ABSTRACT
}

The elderly are part of a society defined by technology acceleration, communication and information. These transformations happen at a rapid pace, making a big impact in the life of senior citizens. Our premise is to consider older people as active subjects in full exercise of their rights, to whom digital inclusion is also a part of social inclusion. The objective of this paper is, on the one hand, to carry out a content analysis of the policies implemented in Argentina regarding digital inclusion, including: level of coverage and survey of possible existing gaps. On the other hand, data from a national survey on access to resources and use of information and communication technologies by the elderly are analyzed. The conclusions show that there are significant gaps in both access to resources and frequency of use. Regarding national public policies on the subject, we observed that the pandemic showed that there was no strategic plan for the achievement of a policy of inclusion of the elderly in the new technologies.

Keywords: Older people, ICT, Argentina, public policies, programs. 


\section{INTRODUCCIÓN}

En el contexto latinoamericano, existe un franco incremento de la población mayor, y Argentina se encuentra entre los cuatro países más envejecidos de la región (Huenchuan, 2018; Gragnolati et al., 2014). Dentro de los efectos de la transición demográfica, destacamos no solo el aumento de la sobrevida ${ }^{1}$, sino también una mayor demanda de cuidados, junto a un incremento en la inserción de las mujeres en el mercado de trabajo y un descenso en la fecundidad (Magnus, 2009). La conjunción de las características del envejecimiento poblacional sumada a las particularidades en materia económica y de acceso a derechos básicos que presenta cada país - muestra una diversidad y desigualdad entre los países de la región. Esta diversidad se ve también reflejada, por ejemplo, en cuanto a la educación a lo largo de toda la vida. En particular, subrayamos el acceso y apropiación de las nuevas tecnologías por parte de la población mayor. Sobre las brechas de acceso a las tecnologías de información y comunicación TIC, justamente, el lema elegido por las Naciones Unidas para celebrar el día internacional de las personas de edad en 2021 es el logro de una equidad digital sin distinción de edades ${ }^{2}$.

Las personas mayores ${ }^{3}$ se encuentran insertas en una sociedad caracterizada por la aceleración tecnológica, la comunicación y la información, cuyas transformaciones cambian a un ritmo vertiginoso. En este contexto, la inclusión digital se convierte en una vía de acceso a la inclusión social. Una de las maneras de materializar esta inclusión es a través de los programas y políticas. Si consideramos

\footnotetext{
Se refiere al incremento de la esperanza de vida.

Ver: https://www.un.org/es/observances/older-persons-day

En este trabajo se considera «persona mayor» a aquellas que tienen sesenta y más años siguiendo los lineamientos de la Asamblea Mundial de Envejecimiento de 1982, en concordancia con la Convención Interamericana sobre la Protección de los Derechos Humanos de las Personas Mayores.
} 
la política pública como un instrumento de cambio desde una perspectiva social, se asume que esta busca ampliar derechos y mantener e incrementar la autonomía de la población mayor.

El aislamiento social, preventivo y obligatorio fue una medida excepcional que el Estado argentino adoptó en un contexto crítico generado por la pandemia de COVID-19, decretada el 11 de marzo de 2020 por la Organización Mundial de la Salud - OMS, con el objetivo de proteger la salud pública, según el Plan Operativo de Preparación y Respuesta al COVID-19. El 3 de marzo se detectó el primer caso de COVID-19 en Argentina y el Gobierno Nacional consideró que, «atendiendo a las recomendaciones de los organismos internacionales especializados y de los expertos locales, y a la luz de la experiencia de otros países, se determinó que la mejor forma que tenemos para enfrentar la propagación del virus y así cuidar la salud de todas y todos es el aislamiento» ${ }^{4}$ que se instala a partir del 20 de marzo de 2020 en la República Argentina. En este sentido, se dispuso que todas las personas que habitasen en el país o se encontraran en el territorio nacional en forma temporaria deben permanecer en sus domicilios habituales, realizando desplazamientos mínimos.

Este suceso que impactó en la vida cotidiana de las personas mayores puso en evidencia la diversidad de situaciones en el acceso y uso de las TIC por parte de los ciudadanos para resolver cuestiones bancarias, sanitarias o de sociabilidad.

En este contexto nos preguntamos: ¿cuál es la situación de las personas mayores en relación con el acceso a internet y a las TIC? ¿Poseen computadora y saben utilizarla? ¿Acceden a las redes bancarias digitales? ¿Pueden gestionar turnos y comunicarse con su médico y servicios de salud a través de la TIC o la telefonía celular? ¿Qué características tienen estas personas que acceden y las que no lo hacen? ¿Cuáles son los programas que el Estado ofrece para facilitar el acceso a las TIC para las personas mayores?

A partir de estas preguntas básicas, y en el marco de nuestro propio aislamiento, el objetivo de este trabajo fue caracterizar a las personas mayores con relación al acceso a las TIC y relevar revelar las políticas y programas estatales que pudieran facilitar este acceso - o no- a las personas de edad.

En síntesis, nos centramos en el análisis de aquellas políticas que vinculan el acceso a las TIC por las personas mayores o que están destinadas a ellas. Para ello se realizó un análisis de las políticas públicas implementadas como

4 Decreto de Necesidad y Urgencia 260/2020. 
respuesta frente a los desafíos de la pandemia en el uso de nuevas tecnologías en la población mayor.

Se analizaron tres ejes que vinculan el uso de las TIC en la vida de las personas mayores. El primero de ellos se focaliza en la utilización de las tecnologías que los servicios sanitarios implementaron a partir de la pandemia por COVID-19; el segundo reseña el papel de las TIC en el manejo de banca electrónica, y el último expone resultados sobre uso de dispositivos y acceso a internet en la población mayor.

Para caracterizar a las personas mayores urbanas de Argentina con relación a la posesión y uso de computadora, telefonía celular y acceso a internet, procesamos las respuestas a las preguntas específicas que integran la encuesta ENES|PISAC a través del programa SPSS. Se realizó, además, un relevamiento por internet de las políticas públicas destinadas a las personas mayores y se analizó su contenido (Bardin, 2002; Krippendorff, 1990), teniendo en cuenta objetivos del plan o programa, población objetivo, lineamientos centrales e implementación.

\section{DERECHOS Y ACCESO A TIC}

El enfoque de derechos humanos promueve su titularidad para todos los grupos sociales (Huenchuan, 2018). De ese modo, contribuye a que aquellas personas que en el pasado fueron excluidas sean tratadas sobre la base de la igualdad y el respeto por la dignidad humana. De acuerdo con este enfoque, se busca asegurar el ejercicio pleno de todos los derechos civiles, políticos, económicos, sociales y culturales de las personas mayores (OEA, 2011). Desde esta perspectiva, uno de los desafíos centrales de las políticas públicas es determinar la forma de contribuir a la construcción de una sociedad con lugar para todos y todas.

Con el fin de lograr una declaración sobre los derechos de las personas mayores, han existido diferentes iniciativas presentadas formalmente o discutidas como documentos en los organismos de las Naciones Unidas y sus respectivos órganos especializados.

En 1982, los Estados miembros de las Naciones Unidas adoptaron el «Plan de Acción Internacional de Viena» en la Primera Asamblea Mundial sobre el Envejecimiento, realizada en Austria. Los Estados que asistieron a la asamblea

[...] reafirmaron su creencia en que los derechos fundamentales e inalienables consagrados en la Declaración Universal de los Derechos Humanos se aplican plenamente y sin menoscabo a las personas de edad y reconocieron 
que la calidad de vida no es menos importante que la longevidad y que, por consiguiente, las personas de edad deben, en la medida de lo posible, disfrutar en el seno de sus propias familias y comunidades de una vida plena, saludable y satisfactoria y ser estimados como parte integrante de la sociedad (Naciones Unidas, 1982).

Las recomendaciones internacionales respecto de la protección de los derechos de las personas mayores han promovido la adecuación de las políticas a estos marcos normativos. En línea con estas recomendaciones, en 1991 se declaran los Principios de las Naciones Unidas en favor de las Personas de Edad ${ }^{5}$, luego retomados en la Convención Interamericana sobre la Protección de los Derechos Humanos de las Personas Mayores. Este instrumento compromete a los Estados a:

promover, proteger y asegurar el reconocimiento y el pleno goce y ejercicio, en condiciones de igualdad, de todos los derechos humanos y libertades fundamentales de la persona mayor, a fin de contribuir a su plena inclusión, integración y participación en la sociedad (Convención Interamericana sobre la Protección de los Derechos Humanos de las Personas Mayores, adoptada el 15 de junio de 2015).

Es importante destacar que esta es una de las convenciones más holísticas en el mundo, pues contempla casi todos los aspectos de la vida de las personas mayores. De este modo, sirve como mecanismo integrador de los principios y derechos señalados en la serie de instrumentos sobre la materia que se han promulgado en el sistema interamericano y el sistema universal de protección de los derechos humanos. Con respecto al ejercicio de la ciudadanía, la convención protege y reconoce el derecho a la educación que, en su artículo 20 prevé:

d) Promover la educación y formación de la persona mayor en el uso de las nuevas TIC para minimizar la brecha digital, generacional y geográfica e incrementar la integración social y comunitaria ${ }^{6}$ (Convención Interamericana sobre la Protección de los Derechos Humanos de las Personas Mayores, adoptada el 15 de junio de 2015).

Como hemos visto esto funge como marco normativo en donde las nuevas tecnologías no deberían convertirse en una barrera para la inclusión tecnológica y social de las personas mayores.

Independencia, participación, cuidados, autorrealización y dignidad.

Destacamos que en Argentina la convención se sancionó y ratificó con fuerza de Ley en 2017

(Ley 27360, Boletín oficial, 2017, mayo). 


\section{LAS TIC Y LAS PERSONAS MAYORES}

La aceleración parece ser un rasgo constitutivo de la modernidad. Esta sensación se experimenta en muchos procesos económicos, sociales, culturales y tecnológicos. La aceleración social actúa por oleadas en muchos casos originadas por las nuevas tecnologías. Para Rosa (2011), la aceleración de las sociedades occidentales se explica a partir de tres procesos que están impulsados, a su vez, por tres ejes: la aceleración tecnológica, el cambio social y el ritmo de vida. Los cambios tecnológicos gozan de una gran velocidad, sobre todo, en torno a las comunicaciones y transporte. En cuanto al cambio social, el autor se refiere específicamente a las trayectorias familiares y laborales, indicando que no hay una estabilidad que las caracterice sino más bien todo lo contrario. La aceleración del ritmo de vida alude a la velocidad con que se suceden las experiencias en la vida diaria, es decir, realizamos muchas más acciones en menos tiempo. Los motores que impulsan este proceso son el económico (el capitalismo), cultural (aceleración de las experiencias vividas) ${ }^{7}$ y estructural (abundancia de opciones y posibilidades).

Para comprender los cambios y transformaciones que vinculan nuevas tecnologías y envejecimiento, nos focalizamos en la aceleración tecnológica definida como la relación de los procesos intencionales dirigidos a acelerar el transporte, la comunicación y la producción de datos, procesos todos que se han incrementado significativamente (Rosa, 2011). Esto también supone una compresión temporal, debido al predominio del tiempo sobre el espacio propiciado por la conectividad vía internet en diversas latitudes que transforma la orientación espacial.

Según Rosa (2016), la aceleración tecnológica promueve una serie de prácticas sociales y modelos de interacción social que afectan las formas en las cuales se construye la identidad. Si asumimos que la aceleración tecnológica cambia a un ritmo vertiginoso, cabe preguntarse cómo es esto afrontado por las diferentes generaciones y, en particular, por las personas mayores. En la «burbuja tecnológica», quedarse parado implica quedar fuera, por eso las personas se ven compelidas y presionadas a mantener el ritmo de cambio social para evitar la pérdida de conexiones valiosas. Consideramos que las tecnologías de la información y comunicación son parte de la vida cotidiana de todas las personas.

Esto incluye la coexistencia de «mosaicos temporales» donde conviven grupos como las personas mayores o los desempleados que, muchas veces, se ven impulsados a desacelerar. 
El desarrollo de las TIC a lo largo del siglo XXI ha dado lugar al surgimiento de la «sociedad de la información» que, según Carmona y Fuentealba (2018), implica la aceleración del proceso de comunicación y el mayor acceso a la información. Las TIC se definen como «aquellas tecnologías que permiten la adquisición, almacenamiento, procesamiento, evaluación, transmisión, distribución y difusión de la información» (Sánchez et al., 2012, p. 121). Suponen la convergencia de la informática, las telecomunicaciones, la electrónica y la microelectrónica. Entre sus características más relevantes encontramos que tienen gran capacidad de penetración e influencia en la economía y en la sociedad toda. Según Castells (2004), las nuevas tecnologías tienen la capacidad de procesar, recombinar y distribuir grandes volúmenes de información. Las TIC fueron incorporándose a un sinfín de actividades y acciones propias del cotidiano de la población, lo que implicó la necesidad de aprender y apropiarse de estas innovaciones para poder desplegarse y vivir en esta nueva sociedad. Es decir, es esta estructura, dinámica y cambiante, la que genera que el ser humano se encuentre en un constante proceso de aprendizaje. Para Boarini et al. (2006), este proceso de incorporación de las TIC inscribe a las personas en un medio de alta densidad comunicativa.

Sin embargo, debe considerarse que no todos los procesos de aprendizaje son similares y se desarrollan a un mismo ritmo en los grupos sociales. Esto es más claro en las personas mayores, dado que tienen que adaptarse a una «nueva sociedad» cuando han tenido poco contacto con las TIC. Así, esta franja de la población debe aprender a vivir diariamente en una sociedad caracterizada por la comunicación, la información y la tecnología. La incorporación de las TIC en las distintas esferas de la vida cotidiana representa un nuevo desafío para la población adulta mayor, sobre todo en lo que respecta a la promoción de su autonomía.

La idea de brecha digital se utilizó en sus inicios para destacar la dificultad que tanto países, grupos sociales como personas tenían en torno al acceso a las tecnologías digitales (Selwyn y Facer, 2007). Se define, a su vez, por la desigualdad en el acceso y uso de las tecnologías y de internet entre las diferentes generaciones (Boonaert y Vetterburg, 2011). Si bien partimos de considerar que existe una diversidad dentro del colectivo de personas mayores, el análisis del uso y acceso a las TIC es clave para determinar factores que agudizan brechas y desigualdades dentro de la población mayor. Así, el concepto de «nativos» o «inmigrantes» digitales nos provee una pista interesante para el análisis (Prensky, 2010), pues mientras que los primeros han nacido en un medio donde utilizan cotidianamente el lenguaje digital ya sea a través de juegos, internet o computa- 
doras, los segundos han tenido que adaptarse y aprender este nuevo lenguaje. No obstante, es importante advertir que no se trata solo de una diferencia de edad entre jóvenes y viejos que actúa como único determinante en la apropiación de las TIC. En efecto, existen otros aspectos que también influyen en el uso y aprendizaje de las TIC como la representación que tienen las personas de ellas, los beneficios que les atribuyen a partir de su uso cotidiano, la complejidad de las interfaces y dispositivos, así como la motivación y el interés por aprender (Rivoir, 2019).

Debido al incremento de la utilización de las TIC, por un lado, y al aumento de la población envejecida, por otro, surgieron muchos estudios e investigaciones interesadas en analizar cómo es el uso de las TIC y sus implicancias en este segmento poblacional. Por su parte, el uso, el derecho al aprendizaje y el acceso a las TIC se encuentra también relacionado con el ejercicio de la ciudadanía (Montijano Cabrera et al., 2010), se trata de una dimensión transversal para la integración social de la población adulta mayor al medio social (Casamayou y González, 2017).

Un estudio comparativo entre Francia, Reino Unido y España destaca el hecho de que el uso de las TIC representa una moda y una tendencia de las cuales las personas mayores participan ampliamente, sobre todo en los medios anglosajón y francófono (González-Oñate et al., 2015). Los franceses poseen un amplio conocimiento sobre las nuevas tecnologías y las redes sociales, e incluso a la hora de no comprender algún aspecto recurren a tutoriales. De esta forma, las TIC constituyen un enlace con el mundo exterior, un medio de estar y permanecer integrado en la sociedad francesa. En cuanto al Reino Unido, el conocimiento relativo a las TIC fue menor. No obstante, la población británica mostró interés por aprender e incorporar estos conocimientos, dado que son conscientes de cómo aquellas pueden incidir en el mejoramiento de la calidad de vida. En lo que respecta a los españoles, el uso de las tecnologías fue menor en comparación con los otros dos países. Esto se acentúa en los sectores sociales más bajos, donde se encontró mayor reticencia en la disposición al aprendizaje de las nuevas tecnologías.

Una investigación comparativa entre veintiocho países que componen la Unión Europea (UE) buscó indagar sobre el uso y acceso a las TIC de las mujeres mayores. Se utilizaron fuentes secundarias de la Oficina Europea de Estadística. Los países con niveles más altos de utilización de las TIC en personas mayores fueron Luxemburgo, Holanda y Dinamarca, mientras que Bulgaria, Croacia y Rumanía presentaron los menores porcentajes de uso del ordenador (Arias Fernández et al., 2018). 
El trabajo de Martínez (2021) analiza, a partir de una revisión bibliográfica y un análisis de encuestas del Instituto Nacional de Estadísticas en España, cómo es el uso de las tecnologías en la población mayor. Los resultados obtenidos demuestran que las personas de entre 65 a 74 años se encuentran en situación de desuso de tecnologías informáticas y acceso a internet de manera muy acusada en todas comunidades autónomas del país. Este panorama permite afirmar que, en contexto de pandemia, se acentúa el aislamiento social, lo que impacta sin dudas tanto en el acceso a recursos como en la cobertura de necesidades.

En el ámbito regional, un estudio reciente muestra que existe una brecha digital entre los distintos grupos etarios de la población, principalmente entre aquellos mayores de sesenta años (Sunkel y Ullmann, 2019) ${ }^{8}$. Esto indica que hay un determinado porcentaje de la población que no está haciendo uso de los beneficios que traen aparejadas las TIC. Una de las conclusiones del estudio indica que, a mayor edad, disminuye el uso de internet en general. Otros aspectos a destacar son la presencia de hijos como promotores del uso de las TIC, la residencia en zonas urbanas y un mayor nivel de educación formal alcanzado dentro del perfil de usuarios de internet.

El trabajo de Barrantes y Ugarte (2019) analiza el uso y acceso a internet y dispositivos en Lima, Perú y el Área Metropolitana de Buenos Aires, Argentina. Destacan, por un lado, que el capital cultural es un factor clave para el uso y apropiación de internet, y por otro, la presencia de un contacto fluido con redes familiares. Observan un menor uso y acceso en los sectores más bajos y en los que prima la informalidad laboral.

En lo que sigue desplegamos el análisis de las políticas implementadas en Argentina antes y durante la pandemia por COVID-19, lo que incluye el nivel de alcance, cobertura y brechas existentes en diferentes actividades mediatizadas por el uso de TIC. Luego se presentan las conclusiones finales, de las que destacamos, por una parte, la existencia de diferencias tanto en el acceso a recursos como en la frecuencia de uso. Por otra parte, observamos, en cuanto a las políticas públicas nacionales, la ausencia de un plan estratégico con lineamientos concretos que se focalicen en el tema.

8 La investigación referida se centró en datos de encuestas de hogares de ocho países de la región: Chile, Ecuador, El Salvador, Honduras, México, Paraguay, Perú y Uruguay, con el objetivo de analizar el uso de medios digitales y el papel que estos estos desempeñan en la inclusión y calidad de vida de las personas mayores. 


\section{LOS SISTEMAS DE INFORMACIÓN DE SALUD Y CIBERSALUD (HEALTH)}

El uso de las TIC en la asistencia sanitaria, o cibersalud, constituye una herramienta esencial para transformar el sistema sanitario y los servicios de salud con el fin de ofrecer una atención integrada y centrada en las necesidades de las personas, lo que supone, a su vez, que sean apropiadas para los mayores (Organización Panamericana de la Salud, 2016).

El uso de las TIC es fundamental para integrar y gestionar la atención de las personas mayores y evaluar el impacto de las intervenciones. En efecto, los avances en las TIC se están utilizando en todo el mundo para mejorar el acceso a la asistencia sanitaria, así como su calidad y seguridad para garantizar la rentabilidad de la prestación de los servicios de salud. En la Unión Europea, por ejemplo, se estima que, con la introducción de las TIC y de la telemedicina, mejoró en un $20 \%$ la eficacia de la atención de salud.

La cibersalud está cambiando el modo en que se prestan los servicios sanitarios y la forma como funcionan en muchos países. Las historias clínicas electrónicas y los sistemas de información de salud relacionados pueden captar, organizar y compartir información sobre pacientes y poblaciones clínicas, con el fin de ayudar a determinar las necesidades de las personas mayores, planificar la asistencia que recibirán, vigilar las respuestas al tratamiento y evaluar los resultados del proceso de atención. También pueden facilitar la colaboración entre los trabajadores de la salud y los pacientes que se encuentran en distintos ámbitos o puntos geográficos.

Servicios de salud tales como la telemedicina y las consultas a distancia permiten que las personas tengan acceso a conocimientos de diagnóstico y tratamiento que pueden no encontrarse disponibles localmente. De muchas maneras, las TIC se han vuelto fundamentales para el tratamiento eficaz de las enfermedades crónicas y la implementación de la asistencia por equipos de atención a la salud.

La cibersalud se utiliza cada vez más para mejorar la calidad de vida de las personas mayores, ya que les permite la comunicación desde su hogar con el equipo de salud y los servicios sociales y comunitarios. Asimismo, combate la soledad y el aislamiento, apoya la independencia y facilita el manejo que pueden hacer las personas sobre su situación sociosanitaria.

La utilidad de los sistemas de información en salud también se extiende a la vigilancia, evaluación y planificación de políticas y a la mejora en la atención de las personas mayores, idealmente entre los diferentes sistemas de asistencia 
sanitaria y de atención a largo plazo. Para que esto sea posible, no obstante, es necesario acordar y utilizar sistemáticamente indicadores comunes.

Entre sus posibilidades, se incluyen parámetros y dominios de la capacidad funcional (como desnutrición, movilidad reducida, deterioro cognitivo y sensorial), lo que permite una evaluación regular en grupos de personas mayores.

Al respecto, en la República Argentina, a la ya existente Ley N ${ }^{\circ} 26.529$ sobre los «Derechos del paciente, historia clínica y consentimiento informado» se incorpora por aprobación unánime de ambas Cámaras Legislativas la Ley № 27.553/20. Esta tiene por objeto: (i) establecer que la prescripción y dispensación de medicamentos, y toda otra prescripción, puedan ser redactadas y firmadas a través de firmas manuscritas, electrónicas o digitales, en recetas electrónicas o digitales, en todo el territorio nacional, y (ii) establecer que puedan utilizarse plataformas de teleasistencia en salud, en todo el territorio nacional, de conformidad con la Ley $\mathrm{N}^{\circ} 25.326$ de Protección de los Datos Personales y la Ley $\mathrm{N}^{\circ} 26.529$ de Derechos del Paciente.

La Ley $\mathrm{N}^{\circ} 27.553 / 20$ se aprueba en el marco de la pandemia COVID-19, donde rigen medidas de Aislamiento Social Preventivo y Obligatorio - ASPO que limitan, entre otras restricciones, las consultas médicas presenciales. Resulta particularmente importante para el acceso a la medicación y la atención de los adultos mayores, pues incluso pueden gestionarse vía WhatsApp. Al mismo tiempo, permite la prescripción y dispensación electrónica de todo tipo de medicamentos (incluidos los controlados, como estupefacientes y psicotrópicos) a través de la firma electrónica simple o avanzada.

Estas medidas, de reciente implementación, coaccionadas por el contexto de la pandemia, tendrán que ser evaluadas, dado que no se conoce su alcance real. La pandemia pone al descubierto desigualdades estructurales, al tiempo que refleja experiencias dispares influenciadas por los determinantes sociales de la salud $^{9}$ (Bambra et al., 2020).

En el contexto de emergencia sanitaria, observamos una tensión entre la necesidad de preservar del contagio a los mayores y la interrupción o postergación de los controles de afecciones crónicas que mostrará, más temprano que tarde, impactos en la movilidad y en la salud mental y física. No contamos con datos recientes sobre el tipo de acceso a la atención médica remota para las personas mayores, dado que aún estamos transitando la pandemia. Desde el campo de investigación, también identificamos limitaciones para realizar un estudio evaluativo

9 Sobre los determinantes sociales de la salud ver Moral, Gascón y Abad (2014). 
que integre a una muestra representativa de la población mayor, debido a que los datos se recolectan a través de canales digitales (Rocco y Oliari, 2007).

\section{LAS TIC Y BANCARIZACIÓN}

A partir del año 2000 y en la década siguiente las políticas públicas impactaron en el crecimiento de la bancarización de las familias, a través de la transferencia vía sistema bancario de programas sociales específicos, cuentas sueldos y jubilaciones, que pasaron de 10 a 15 millones de usuarios. Sin embargo, la infraestructura bancaria no acompañó al crecimiento de los clientes: en Buenos Aires hubo una baja cantidad de agencias bancarias con alta concentración en la Ciudad de Buenos Aires con relación al resto del país.

Según la Encuesta ENES|PISAC ${ }^{10}$, relevada en el año 2015 y que integró a 8265 hogares y 27610 personas del territorio urbano argentino, el 71\% de las personas entrevistadas afirmó tener acceso a una cuenta bancaria. De ellos, el $42 \%$ tiene una cuenta jubilatoria o sueldo y el $44 \%$, accede además a otro tipo de cuentas. Con respecto a los hogares de los adultos mayores, el $83 \%$ posee una cuenta bancaria, debido a que en Argentina el acceso al sistema jubilatorio se encuentra muy extendido. Sin embargo, las personas mayores tienden a cobrar su jubilación en las escasas entidades bancarias y utilizan poco el sistema de cajeros automáticos y menos aún el sistema online.

En esta misma línea, los datos de la encuesta sobre calidad de vida (EnCaViam, INDEC 2012) muestran que el 64\% de los encuestados no usa por sí solo el cajero, lo que implica una demanda de ayuda a terceros, ya sea para retirar dinero o para realizar trámites por este medio. De ese total, cuatro de cada diez prefieren retirar el dinero por ventanilla y un $21 \%$ considera que su funcionamiento es complicado a pesar de que la mayoría (82\%) cuenta con una tarjeta de débito. En cuanto a las personas mayores de 75 años, encontramos que cerca del $80 \%$ no utiliza su tarjeta de débito. Son las mujeres quienes hacen menor uso del cajero automático.

Por ello, al implementarse el Aislamiento Social Preventivo y Obligatorio como producto de la política sanitaria para mitigar el COVID-19, se produjo una situación crítica de desborde ante la masividad de presencia de este grupo de personas en las escasas sucursales bancarias (Clarín, Sociedad, 2020, 3 de abril).

10 Encuesta ENES (Encuesta Nacional sobre la Estructura Social), PISAC (Programa de Investigación sobre la Sociedad Argentina Contemporánea). 
Uno de los principales fue que los adultos mayores no sabían usar el sistema de cajeros automáticos ni el home banking.

En respuesta a esta situación, se implementó una nueva política estatal a partir del programa «Fábrica, Diseño e Innovación» del Consejo Federal de Ciencia y Tecnología - COFECyT, en articulación con la Dirección General de Protección y Desarrollo Sostenible de la Secretaría de Integración Social de Personas Mayores del Gobierno de la Ciudad de Buenos Aires. Se implementó en esta jurisdicción una prueba piloto del taller «Yo digital». Este programa está diseñado para que las personas mayores hagan uso independiente del cajero automático para el cobro de sus jubilaciones o pensiones. Los temas abarcados para la formación de formadores, encargados de capacitar a los mayores, son acercamiento a la tecnología de cajeros automáticos; dinámica y enseñanza de pasos para la extracción de haberes; y práctica digital que integra las interfaces del uso de las redes de tarjetas bancarias.

\section{Las personas de mayor edad y el acceso y uso de las TIC}

Según la mencionada encuesta ENES|PISAC, las personas mayores ${ }^{11}$ que poseen computadora en Argentina comprenden al 38\% de los encuestados (1640), en tanto que prácticamente dos terceras partes no cuentan con este recurso $(61,6 \%)$. Las personas que poseen computadora se componen por mujeres en un 55\% (902) y varones en un $45 \%$ (738). Su distribución por edad es para el grupo de 60-69 años del $63,5 \%$, entre los de $70-79$ años del $25,9 \%$ y para el grupo de 80 y más años se trata del $10,6 \%$.

Al tener en cuenta el nivel educativo de los mayores que poseen computadora, observamos que representan el $25 \%$ de aquellos que completaron hasta el nivel primario, el $47,3 \%$ de los que culminaron la escuela secundaria y el $64,4 \%$ de las personas mayores que cuenta con estudios en el superiores.

En síntesis, la posesión de computadoras en los hogares estudiados disminuye con el incremento en la edad de la población y en los niveles educativos más bajos.

Al indagar sobre el nivel alcanzado en el manejo de la computadora la distribución de la tabla 1, nos indica que dos terceras partes de la muestra no manejan esta tecnología y que un quinto de esta solo lo hace en el nivel básico. Solo un

11 Para una mayor caracterización de las personas mayores que respondieron la encuesta ENES|PISAC, ver Oddone (2018). 
$4 \%$ de las personas de mayor edad indican tener un conocimiento avanzado en computación.

Tabla 1. Nivel de manejo de computadora

Personas de 60 y más sesenta años o más

\begin{tabular}{ccc}
\hline Nivel & Número & Porcentaje (\%) \\
\hline Sí, nivel básico & 885 & 20,5 \\
Sí, nivel medio & 336 & 7,8 \\
Sí, nivel avanzado & 166 & 3,8 \\
No maneja & 2933 & 67,9 \\
\hline TOTAL & 4320 & 100,0 \\
\hline
\end{tabular}

Elaboración propia a partir de la encuesta ENES|PISAC

En cuanto al nivel de uso de computadora con relación al tipo de hogar en el que residen los adultos mayores argentinos, vemos que aquellos que viven con hijos tienen un mejor desempeño en el manejo de este dispositivo en todos los niveles con respecto a los que viven solos o en pareja (ver tabla 2). Esto nos está indicando la importancia que adquiere el intercambio entre las generaciones para una facilitación del uso de las TIC en la gente mayor, de modo que así disminuye la brecha digital (Sunkel y Ullmann, 2019; Barrantes y Ugarte, 2019).

Tabla 2. Nivel de manejo de computadora por tipo de hogar

Personas de sesenta y más años

\begin{tabular}{ccccc}
\hline Nivel & $\begin{array}{c}\text { Unipersonal } \\
(\mathbf{\%})\end{array}$ & $\begin{array}{c}\text { Nuclear sin } \\
\text { hijos (\%) }\end{array}$ & $\begin{array}{c}\text { Nuclear con } \\
\text { hijos (\%) }\end{array}$ & $\begin{array}{c}\text { Nuclear }^{\mathbf{1 2}} \\
\text { incompleto (\%) }^{(\%)}\end{array}$ \\
\hline Sí, nivel básico & 18,4 & 23,0 & 28,8 & 18,2 \\
Sí, nivel medio & 8,6 & 10,3 & 10,6 & 5,9 \\
Sí, nivel avanzado & 4,2 & 3,6 & 9,0 & 4,5 \\
No maneja & 68,8 & 63,1 & 51,6 & 71,4 \\
\hline TOTAL & 100,0 & 100,0 & 100,0 & 100,0 \\
\hline
\end{tabular}

Elaboración propia a partir de la encuesta ENES|PISAC.

12 Se refiere jefe o jefa de hogar sin cónyuge y con hijos e hijas. 
Sobre este tema destacamos que Barrantes y Cozzubo (2015) analizan variables sociodemográficas tales como género, edad, nivel educativo e ingreso, y trabajan sobre la base de datos de la Encuesta sobre el uso de internet (2014) en Buenos Aires, Lima y Guatemala. Su análisis descriptivo muestra la prevalencia de la brecha generacional en América Latina. Al estudiar la influencia que la composición familiar puede tener en la adopción y la intensidad de uso de internet por adultos mayores en América Latina, comprueban con el análisis de regresión que se da el aprendizaje intergeneracional en las relaciones entre menores de edad y adultos mayores dentro del hogar, y un incremento del uso de internet a partir de la convivencia con otras personas mayores.

Con respecto a la telefonía celular, el 78\% de la población de personas mayores cuenta con esta tecnología, mientras que un $21,5 \%$ no posee un teléfono de estas características. Este $78 \%$ se compone de varones en un $44 \%$ y de mujeres en el $56 \%$ de los casos. Su distribución por edad es para el grupo de 60-69 años del $58,4 \%$, para el de $70-79$ años del 30,6, y para el grupo de 80 y más años se trata del $11,1 \%$.

Si se considera el nivel educativo de aquellos mayores que usan telefonía celular, vemos que un $71,1 \%$ completó hasta la escuela primaria, la escuela secundaria fue realizada por el $82,7 \%$ y el nivel superior fue alcanzado por el $90,6 \%$.

Resultan ilustrativos los datos que brinda la encuesta EnCaViam (INDEC, 2012) en relación con las personas de mayor edad que no usan telefonía celular, porque aducen que no les interesa usar esa tecnología, que les resulta complicada, o bien que tienen algún impedimento de tipo físico, como que no escuchan bien, no ven bien los números o no pueden manipular este dispositivo.

En cuanto al uso de internet, los datos aportados por el módulo de acceso y uso de tecnologías de la información y comunicación de la Encuesta Permanente de Hogares (cuarto trimestre, INDEC, 2018) indican que las personas mayores de 65 años que hicieron uso de internet en los últimos tres meses anteriores a la encuesta fueron cuatro de cada diez. Esta proporción se incrementa cuando las personas alcanzan niveles de escolaridad más elevados.

En este contexto, no es menor la importancia que cobran las políticas y programas existentes dirigidos a la inclusión digital de las personas mayores.

El programa de «postas digitales», dependiente de la Dirección Nacional de Políticas para Adultos Mayores del Ministerio de Desarrollo Social de la Nación, se destina a adultos mayores que asisten a cuatro clases mensuales, una vez por semana, con una carga horaria de tres horas, con el fin de eliminar la brecha digital 
en este grupo de edad ${ }^{13}$. El programa se implementa en el territorio argentino a través de las provincias, municipios u ONG.

Otro de los programas que incluye cursos de alfabetización digital es el programa Universidad para Adultos Mayores Integrados (UPAMI) del Instituto Nacional de Servicios Sociales para Jubilados y Pensionados (INSSJP-PAMI) ${ }^{\mathbf{1 4}}$. Este espacio universitario está destinado a los afiliados a esta obra social cuyos objetivos son: efectivizar la participación y el acceso a la educación de las personas mayores, mejorar la calidad de vida y promover la igualdad de oportunidades para el desarrollo de valores culturales y vocacionales. Además, fomenta la educación continua a lo largo de la vida, permitiendo la inserción de las personas adultas mayores, a la vez que significa un intercambio cultural e intergeneracional en el que se recuperan y valoran saberes personales y sociales. En este marco, se ofertan talleres de actualización tecnológica para todos los afiliados del país. Se accede a través de las universidades que forman parte del programa ${ }^{15}$. Con respecto a este último programa, cabe aclarar que durante la primera etapa de la pandemia los cursos se suspendieron. Luego se retomaron paulatinamente a través de una planificación que integra las TIC sobre una población focalizada que tenía acceso previo a la conectividad y dispositivos. Destacamos que estos intercambios entre docentes y participantes del programa eran previos a la pandemia (Martínez et al., 2020).

\section{REFLEXIONES FINALES}

Considerando los estudios sobre el acceso y uso de las TIC en personas mayores, hay dos premisas sobre las que se sustenta este trabajo: una busca entender la inclusión digital como una forma de materializar la inclusión social y otra se vincula con el papel que pueden tener las TIC en la profundización de las desigualdades sociales y económicas e incluso en la creación de otras nuevas. Asimismo, destacamos que las valoraciones y usos que las personas mayores hacen de las TIC impactan de lleno en su vida cotidiana, por ejemplo, en la gestión de los recursos financieros, la solicitud de trámites referidos a su salud, la sociabilidad y la comunicación.

13 Fuente: Ministerio de Desarrollo Social de la Provincia de Buenos Aires. Recuperado de https://www.gba.gob.ar/desarrollosocial

14 Instituto Nacional de Servicios Sociales para Jubilados y Pensionados.

15 Instituto Nacional de Servicios Sociales para Jubilados y Pensionados. Recuperado de https:// www.pami.org.ar/inicio 
En Argentina, como hemos observado, la utilización de las TIC atraviesa la vida cotidiana de las personas mayores. Destacamos positivamente que el contexto de pandemia permitió agilizar y dar curso a la Ley de Recetas Electrónicas $27.553 / 20$, lo que contribuyó a la materialización del acceso a la salud. Si bien estas medidas buscan facilitar el cumplimiento del derecho a la salud, no siempre lo logran debido a la existencia de barreras (económicas y generacionales, entre otras) que terminan, muchas veces, reproduciendo y acentuando desigualdades y brechas preexistentes. A su vez, la premura de los cambios que se suscitaron con la pandemia puso en jaque el tiempo necesario para generar un aprendizaje significativo y acorde con las necesidades de las personas mayores. Así, la demanda burocrática de usos variados de plataformas y aplicaciones para realizar trámites, impuestos por la necesidad de respetar la distancia social (permisos de circulación, turnos bancarios, trámites de distinta índole) se convirtió en otra barrera para esta población.

La emergencia sanitaria puso de manifiesto la necesidad de que la población mayor pudiera acceder al sistema bancario a partir de canales tecnológicos, en pos de reducir la exposición al contagio de COVID-19. Esta coyuntura mostró, en lo que refiere al uso del cajero automático, compras online y uso de home banking, que la situación resulta dificultosa para este grupo de edad. La autonomía en el manejo del propio ingreso es un tema sensible y, en este contexto, cercena más el derecho a poder decidir, favoreciendo las situaciones de abuso y maltrato.

En cuanto al uso de dispositivos (computadoras, sobre todo) y el acceso a internet, hemos visto que contar con mayores niveles de educación formal o mantener un contacto fluido con otras generaciones favorece y facilita la inclusión digital. Observamos que las personas mayores utilizan con mayor frecuencia el teléfono celular, recurso clave en el contexto actual.

La investigación nos muestra claramente que la pandemia evidenció la ausencia de un plan estratégico nacional de inclusión digital para las personas mayores, así como la existencia de una brecha digital entre las personas mayores y otras generaciones. Por lo tanto, es deseable que las políticas públicas favorezcan la promoción de la solidaridad y el intercambio generacional, creando lazos participativos que eviten la segregación social y el viejismo y promuevan la transmisión de saberes. 


\section{REFERENCIAS}

Acceso y Uso a las Tecnologías de la Información y Comunicación (MAUTIC) de la Encuesta Permanente de Hogares INDEC (2018). Informe Técnico, 3(86), 1-16.

Arias Fernández, E., Castro Lirio, J., González Alonso, D. et al. (2018). Acceso y uso de las TIC de las mujeres mayores de la Europa comunitaria. Prisma Social: Revista de Investigación Social, (21), 282-315.

Bambra, C., Riordan, R., Ford, J. y Matthews, F. (2020). The COVID-19 pandemic and health inequalities. Journal of Epidemiology and Community Health, 74(11), 964-968. https://doi.org/10.1136/jech-2020-214401

Bardin, L. (2002). Análisis de contenido. Madrid: Akal.

Barrantes, R. M. y Ugarte, D. R. (2019). La apropiación de internet por adultos mayores: desafíos planteados por las economías informales en dos ciudades de América Latina. Research on Ageing and Social Policy, 7(1), 304-333. https:// doi.org/10.17583/rasp.2019.3962

Barrantes, R. y Cozzubo, A. (2015). Edad para aprender, edad para enseñar: el rol del aprendizaje intergeneracional intrahogar en el uso de la Internet por parte de los adultos mayores en Latinoamérica. Documento de trabajo 411. Lima: Departamento de Economía de la Pontificia Universidad Católica del Perú.

Boarini, M. N., Cerdá, E. P. y Rocha, S. (2006). La educación de los adultos mayores en TIC. Nuevas competencias para la sociedad de hoy. Revista Iberoamericana de Tecnología en Educación y Educación en Tecnología, (1), 7.

Boonaert, T. y Vettenburg, N. (2011). Young people's internet use: Divided or diversified? Childhood, 18(1), 54-66. https://doi.org/10.1177/0907568210367524

Carmona, C. B. y Fuentealba, S. C. (2018). Una mirada histórica del impacto de las TIC en la sociedad del conocimiento en el contexto nacional actual. Contextos, Revista de Humanidades y Ciencias Sociales, 41, 5.

Casamayou, A. y Gonzales Morales, M. (2017). Personas mayores y tecnologías digitales: desafíos de un binomio. Revista Psicología, Conocimiento y Sociedad, 7(2), 199-226. https://doi.org/10.26864/PCS.v7.n2.9

Castells, M. (2004). La era de la información: economía, sociedad y cultura (Vol. 1). Buenos Aires: Siglo XXI.

Decreto de Necesidad y Urgencia 260/2020 (marzo, 2020). Emergencia Sanitaria Coronavirus (COVID-19). Boletín oficial de la República Argentina. Recuperado de http://servicios.infoleg.gob.ar/infolegInternet/verNorma.do?id=335423 [consultado el 10 de agosto de 2021]. 
Diario Clarín (2020, 3 de abril). Horas de espera. Coronavirus en Argentina: acampes, bufandas como barbijos y colas interminables para cobrar jubilaciones $y$ planes Recuperado de https:/www.clarin.com/sociedad/coronavirus-argentinaacampes-bufandas-barbijos-colas-interminables-cobrar-jubilaciones-planes_0_ ZFTydvpEC.html [consultado el 12 de diciembre de 2020].

Encuesta ENES (Encuesta Nacional sobre la Estructura Social) PISAC (Programa de Investigación sobre la Sociedad Argentina Contemporánea). Recuperado de http://pisac.mincyt.gob.ar/ [consultado el $1^{\circ}$ de septiembre de 2020]

González-Oñate, C., Fanjul-Peyró, C. y Cabezuelo-Lorenzo, F. (2015). Uso, consumo y conocimiento de las nuevas tecnologías en personas mayores en Francia, Reino Unido y España. Comunicar, 22(45), 19-28. https://doi.org/10.3916/C45-201502

Gragnolati, M., Rofman, R., Apella, I. y Troiano, S. (2014). Capítulo I. En M. Gragnolati, R. Rofman, I. Apella y S. Troiano (eds.), Los años no vienen solos. Oportunidades y desafíos económicos de la transición demográfica en Argentina (pp. 13-51). Uruguay: Banco Mundial.

Huenchuan, S. (2018). Envejecimiento, personas mayores y Agenda 2030 para el Desarrollo Sostenible: perspectiva regional y de derechos humanos. Cepal. https:// doi.org/10.18356/19532890-es

Krippendorff, K. (1990). Metodología de análisis de contenido teoría y práctica. Barcelona: Paidós.

Ley $\mathrm{N}^{\circ} 27.553 / 20$ Recetas electrónicas o digitales (2020, 11 de agosto). Boletín Oficial de la República Argentina. Recuperado de https://www.boletinoficial.gob.ar/ detalleAviso/primera/233439/20200811 [consultado el 10 de agosto de 2020].

Ley Nacional № 27360 - Convención Interamericana sobre Protección de los Derechos Humanos de las Personas Mayores (2017, 31 de mayo). Boletín Oficial de la República Argentina. Recuperado de https://www.boletinoficial.gob.ar/detalleAviso/primera/164386/20170531 [consultado el 9 de septiembre de 2020].

Martínez, A. R. (2021). Vejez activa, el reto tecnológico. En Luces en el camino: filosofía y ciencias sociales en tiempos de desconcierto (pp. 2642-2665). Dykinson.

Martínez. G., Herrera, M., Lado, S., Molina, S. y Pochintesta, P. (2020). Resistiendo sin corona ¿cómo construir antídotos comunitarios en cuarentena? Geronte. Revista de Estudios sobre procesos de la vejez, 7(1), 33-40.

Montijano Cabrera, Ma. P., García Mestanza, J., Delgado Peña, J. J. y Domínguez, A. C. (2010) ¿Juegan las TIC un papel importante en la vida de los mayores?: el caso del Aula de Mayores de la Universidad de Málaga. Recuperado de http://www. ecom45.uma.es/images/stories/results-documents/Sevilla-junio-2010-VERSION\%20FINAL.pdf [consultado el 10 de septiembre de 2020]. 
Moral, P. A. P., Gascón, M. L. G. y Abad, M. L. (2014). La salud y sus determinantes sociales. Desigualdades y exclusión en la sociedad del siglo XXI. Revista Internacional de Sociología, 72(1), 45-70. https://doi.org/10.3989/ris.2013.02.16

Oddone, M. J. (2018). Condiciones de vida de las personas mayores. En J. I. Piovani y A. Salvia (coords.), La Argentina en el siglo XXI cómo vivimos y convivimos en una sociedad desigual. Encuesta Nacional sobre la Estructura Social (pp. 593-623). Buenos Aires: Siglo XXI.

Organización de los Estados Americanos (2011). Informe sobre la situación de las personas mayores en el hemisferio y la efectividad de los instrumentos universales y regionales vinculantes de derechos humanos con relación a la protección de los derechos de las personas mayores en el siglo XXI derechos de las personas mayores. Washington, D. C. Recuperado de http://www.oas.org/consejo/sp/cajp/ Personas\%20Mayores.asp [consultado el 10 de septiembre de 2021].

Organización de los Estados Americanos (2015). Convención Interamericana sobre la Protección de los Derechos Humanos de las Personas Mayores, aprobada el 15 de junio de 2015. Recuperado de http://www.oas.org/es/sla/ddi/ [consultado el 10 de agosto de 2021].

Organización Panamericana de la Salud (2016). Marco de Implementación de un Servicio de Telemedicina. Washington, D. C.: OPS, 2016. Recuperado de https://iris. paho.org/handle/10665.2/28413 [consultado el 15 de agosto 2021]

Prensky, M. (2010). Nativos e inmigrantes digitales. Distribuidora Sek.

Rivoir, A. (2019). Personas mayores y tecnologías digitales. En A. L. Rivoir y M. J. Morales (coords.), Tecnologías digitales. Miradas críticas de la apropiación en América Latina (pp. 51-63). Buenos Aires: Consejo Latinoamericano de Ciencias Sociales. https://doi.org/10.2307/j.ctvt6rmh6.6

Rocco, L. y Oliari, N. (2007). La encuesta mediante internet como alternativa metodológica. Trabajo presentado en VII Jornadas de Sociología, Facultad de Ciencias Sociales-Universidad de Buenos Aires, Buenos Aires-Argentina. Recuperado de http://www.aacademica.org/000-106/392

Rosa, H. (2011). Aceleración social: consecuencias éticas y políticas de una sociedad de alta velocidad desincronizada. Persona y Sociedad, 25(1), 9-49.

Rosa, H. (2016). Alienación y aceleración: hacia una teoría crítica de la temporalidad en la modernidad tardía. Buenos Aires: Katz. https://doi.org/10.2307/j.ctvndv5zf

Sánchez-Torres, J. M., González-Zabala, M. P. y Muñoz, M. P. S. (2012). La sociedad de la información: génesis, iniciativas, concepto y su relación con las TIC. Revista UIS Ingenierías, 11(1), 113-128.

Selwyn, N. y Facer, K. (2007). Beyond the digital divide. Opening Education Reports. Bristol: Futurelab. Recuperado de www.futurelab.org.uk/openingeducation 


\section{Páginas web consultadas}

Instituto Nacional de Servicios Sociales para Jubilados y Pensionados: https://www. pami.org.ar/inicio [consultado el 11 de agosto de 2020].

Ministerio de Ciencia Tecnología e innovación: https://www.argentina.gob.ar/noticias/ inclusion-digital-para-personas-mayores [consultado el 20 de agosto de 2020].

Ministerio de Desarrollo Social de la Provincia de Buenos Aires: https://www.gba.gob. $\mathrm{ar} /$ desarrollosocial [consultado el 20 de agosto de 2020].

Ministerio de Educación de la Nación: https:/www.educ.ar/ recursos/154311?from=151412 [consultado el 10 de septiembre de 2020].

Ministerio de Justicia y Derechos Humanos: https://www.argentina.gob.ar/justicia/ derechofacil/leysimple/covid-19-recetas-medicas [consultado el 10 de agosto de 2020].

Ministerio de Salud y Desarrollo Social de la Nación: https://www.argentina.gob.ar/ justicia/derechofacil/aplicalaley/historia-clinica [consultado el 10 de agosto de 2020]. 